\title{
Low serum testosterone and increased diastolic ocular perfusion pressure: a risk for retinal microvasculature
}

\author{
Nico T. Malan ${ }^{1}$, Wayne Smith ${ }^{1}$, Roland von Känel, Mark Hamer ${ }^{3}$, Alta E. Schutte ${ }^{1,4}$, and Leoné Malan ${ }^{1,4}$ \\ ${ }^{1}$ Hypertension in Africa Research Team, Faculty of Health Sciences, North-West University, Potchefstroom, \\ South Africa \\ ${ }^{2}$ Department of Psychosomatic Medicine, Clinic Barmelweid, Barmelweid, Switzerland \\ ${ }^{3}$ Department of Epidemiology and Public Health, University College of London, UK \\ ${ }^{4}$ MRC Research Unit for Hypertension and Cardiovascular Disease, North-West University, Potchefstroom, \\ South Africa
}

\begin{abstract}
Summary: Background: Low levels of testosterone in men and changes in retinal microvascular calibre are both associated with hypertension and cardiovascular disease risk. Sex hormones are also associated with blood flow in microvascular beds which might be a key intermediate mechanism in the development of hypertension. Whether a direct association between endogenous testosterone and retinal microvascular calibre exists is currently unknown. We aimed to determine whether testosterone is independently associated with ocular perfusion via a possible association with retinal vascular calibre or whether it plays only a secondary role via its effect on blood pressure in a bi-ethnic male cohort.

Probands and methods: A total of 72 black and 81 white men (28-68 years of age) from the follow-up phase of the Sympathetic activity and Ambulatory Blood Pressure in Africans (SABPA) study were included in this sub-study. Ambulatory pulse pressure and intraocular perfusion pressures were
\end{abstract}

obtained, while metabolic variables and testosterone were measured from fasting venous blood samples. Retinal vascular calibre was quantified from digital photographs using standardised protocols.

Results: The black men revealed a poorer cardiometabolic profile and higher pulsatile pressure $(>50 \mathrm{~mm} \mathrm{Hg})$, intraocular pressure and diastolic ocular perfusion pressure than the white men $(\mathrm{p} \leq 0.05)$. Only in the white men was free testosterone positively associated with retinal calibre, i.e. arterio-venular ratio and central retinal arterial calibre and inversely with central retinal venular calibre. These associations were not found in the black men, independent of whether pulse pressure and ocular perfusion pressure were part of the model. Conclusions: These results suggest an independent, protective effect of testosterone on the retinal vasculature where an apparent vasodilatory response in the retinal resistance microvessels was observed in white men.

Key words: Testosterone, pulse pressure, diastolic ocular perfusion pressure, intraocular pressure, retinal microvascular calibre

\section{Introduction}

It is well established that a reduced testosterone (T) level in men is associated with endothelial dysfunction, an increase in blood pressure (BP), reduced microvascular perfusion and the risk of cardiovascular diseases (CVD) [1, 2]. As T levels tend to decrease with age [1] it may be an important causative factor in the development of hypertension (HT) and other CVD in aging men. We have found that low T levels were associated with hyperkinetic BP responses [3], as well as with subclinical structural arterial wall remodelling [4], suggesting a high sensitivity of arterial vessels to the effects of reduced T levels. Results from different prospective studies indicate that the reduced retinal arteriolar diameter and increased venular diameter are associated both with the risk of developing HT as well as a consequence of HT [5].

Changes in retinal vascular calibre are also associated with other cardiometabolic diseases like diabetes, coronary artery disease and stroke [5]. Reduction in arterio-venular ratio (AVR) and central retinal arterial equivalent (CRAE) is normally associated with hypertension risk, whereas an increase in central retinal venular equivalent (CRVE) is associated with stroke risk [6]. Intraocular pressure (IOP) and ocular perfusion pressure (OPP) have also been associated with changes in BP and retinal microvascular calibre [2, 7]. An increase in IOP and/or a decrease in diastolic ocular perfusion pressure (DOPP, calculated as the difference between IOP and DBP) is strongly associated with the development of open angle glaucoma [8].

It is further known that higher levels of $\mathrm{T}$ will tend to increase IOP, thereby decreasing ocular blood flow in menopausal women [2]. However, no information is available regarding the effect of $\mathrm{T}$ on IOP or ocular perfusion pressure in men $[2,9]$. There is evidence that systemic BP fundamentally influences the ocular circulation [9], which may indicate that $\mathrm{T}$, at the least in an indirect way, is associated with retinal vascular perfusion, the main determinants of which are 
DOPP, IOP, BP and retinal microvascular calibre. $T$ is inversely associated with BP (including PP [10]), while BP is inversely associated with arteriolar calibre in the retina [5], raising the possibility of an indirect association between $\mathrm{T}$ and retinal microvascular calibre. In support of this reasoning it has been shown that $\mathrm{T}$ induces vasodilation in mesenteric microvascular resistance vessels [11], but, to the best of our knowledge, whether this is also true for retinal resistance microvessels has thus far not been shown [2]. The main aim of this study was to determine whether serum $\mathrm{T}$ is independently associated with ocular blood flow via a possible association with retinal vascular calibre or whether it plays only a secondary role via its effect on pulse pressure (PP) in a male cohort. We also aimed to determine whether these possible associations were apparent in both ethnic groups.

\section{Probands and methods}

\section{Design and participants}

The protocol and methodology used in this study has been described extensively elsewhere [12].

Participants were initially recruited as part of phase 1 of the prospective Sympathetic activity and Ambulatory Blood Pressure in Africans (SABPA) study conducted between late summer and late autumn of 2007 and 2008. The study sample comprised of urban black and white teachers working in the Kenneth Kaunda Education district in the North West Province of South Africa. The motivation for this selection was to obtain a homogenous sample from a similar working environment and socio-economic status although cultural differences could not be excluded.

We invited all participants of the first phase of SABPA to participate in this follow-up phase of the study conducted between late summer and late autumn of 2011 and 2012. Men are more prone to the development of cardiovascular disease (CVD); therefore, only men were included in order to obtain a homogenous high CVD risk cohort [13].

Of the initial 204 male participants in the first phase, 180 men reported for the second phase.

We included only the second phase participants in this sub-study as no retinal blood vessel measurements were taken during the first phase. Participants with epilepsy $(\mathrm{N}=1)$ and unsuccessful retinal vessel recordings $(\mathrm{N}=26)$ were excluded, leaving a subsample of 153 (72 black and 81 white men). Participants were fully informed about the objectives and procedures of the study prior to their recruitment. All participants provided written, informed consent. The study conformed to the Helsinki Declaration (2007) and was approved by The Ethics Review Board of the North-West University, Potchefstroom Campus: Approval number 0003607 S6.

\section{Assessment of health behaviour}

Participants were instructed to carry on with their habitual daily activities wearing the Actiheart physical activity monitor at all times whilst awake and asleep for a 7 day period. They spent the night in the Metabolic Research Facility of the North-West University in Potchefstroom, South Africa. On Day 2, participants were in a semi-recumbent position from 0715 $\mathrm{h}$ for at least 2 hours during which the 12-lead ECG (NORAV PC 1200) registration was performed followed by blood sampling. Physical activity was assessed with Actiheart (GB0/67703, CamNtech Ltd., Cambridgeshire, UK) monitors. The 12-lead ECG resting heart rate was used to calculate the sleep heart rate required by the Actiheart program when the device was fitted to each participant. Participants were instructed to carry on with their habitual daily activities wearing the monitor at all times whilst awake and asleep for a $24 \mathrm{~h}$ period. Smoking status was assessed using cotinine [14]. To separate smokers from non-smokers, the cotinine values were dichotomised with a cut-off value of $50 \mathrm{ng} / \mathrm{ml}$ [15]. Gamma-glutamyltransferase ( $\gamma$-GT) was measured and may be considered a marker of alcohol consumption [16].

\section{Anthropometric measurements}

All anthropometric measurements were performed in triplicate by registered level II anthropometrists according to standardised procedures. Body surface area (BSA) was calculated based on the Mosteller formula [17]. Intra- and inter-observer variability was less than $5 \%$.

\section{Cardiovascular measurements}

On the morning of the first clinical assessment day (Day 1), ABPM and 2-lead electrocardiograph monitors were attached to participants on the non-dominant arm at their workplace between $0700 \mathrm{~h}$ and $0730 \mathrm{~h}$ (Meditech CE120 CardioTens; Meditech, Budapest, Hungary). The successful inflation rate over this period was $85.8 \%( \pm 9.14)$ in black and $90.4 \%$ $( \pm 8.61)$ in white men. Hypertensive status and CVD risk was classified from $24 \mathrm{~h} \mathrm{ABPM}$ as SBP $\geq 130 \mathrm{~mm}$ $\mathrm{Hg}$ and/or $\mathrm{DBP} \geq 80 \mathrm{~mm} \mathrm{Hg}$ [18]. The ABPM apparatus was removed after the last BP measurement at $0730 \mathrm{~h}$ on Day 2. We additionally calculated ambulatory PP as it has a greater effect on arterial function and, together with retinal perfusion pressure, are associated with the progression of diabetic retinopathy $[19,20]$.

\section{Measurement of retinal vascular calibre}

Static retinal microvascular measurements were performed in a wellcontrolled light and temperature regulated laboratory using an Imedos 
Retinal Vessel Analyser (Germany) with a Zeiss FF450 ${ }^{\text {Plus }}$ camera and the VesselMap 1 Version 3.10 software. No intake of food or caffeine containing beverages, alcohol, smoking or exercise was allowed one hour prior to retinal vessel measurements. Participants were introduced to the procedure and screened for acute anterior angle chamber glaucoma risk with a small light source by a trained registered nurse. Mydriasis was induced in the right eye of the participant by means of a drop containing tropicamide, $1 \%$ and benzalkonium chloride $0.01 \%(\mathrm{~m} / \mathrm{v})$. In the event of previous injury to the right eye, the left eye was used (black men $\mathrm{N}=3$; white men $\mathrm{N}=1$ ). Retinal vascular calibre was measured in the monochrome images by manually selecting first order vessel branches in a measuring zone located between 0.5 and $1.0 \mathrm{op}-$ tic disc diameters from the margin or the optic disc. Upon selection of the vessel, the VesselMap 2, Version 3.02 software automatically delineated the vessels' measuring area. The colour photograph was used as a reference to ascertain correct identification of venules and arterioles. Identification of the vessels was performed by two experienced scientists who had to agree on the vessel type before selection. Automated software calculations, based on the Knudtson revision of the Parr-Hubbard formulas determined estimates from the 6 largest arterioles and venules and were summarised as central CRAE and CRVE, respectively [21]. As the image scale of each eye was unknown, the values of CRAE and CRVE were expressed as measuring units (MU), where $1 \mathrm{MU}$ is equivalent to 1 micrometre when the dimensions of the eye being examined correspond to those of the normal Gullstrand eye. AVR was also calculated (CRAE/CRVE). Reproducibility was computed for a randomly selected cohort with a correlation coefficient of 0.84 . The intra-class correlation (ICC) analysis involved a mixed-model framework, whereby random effects were assumed for subjects and fixed effects were assumed for the graders. The Cronbach's alpha-reliability index for the AVR was 0.91 for this randomized cohort. A registered ophthalmologist diagnosed retinal pathology that can be seen in hypertensive/diabetic retinopathy (i.e., arterio-venular (AV) nicking, and increased optic nerve cup-to-disc ratio indicating optic nerve degeneration). One to two local anaesthetic drops (Novasine Wander 0.4\% Novartis) were inserted in both eyes to measure IOP with the Tono-Pen Avia Applanation Tonometer (Reichert 7-0908, ISO 9001, New York, USA). DOPP was calculated by subtracting the IOP value from the DBP value recorded just prior to measuring the IOP. DOPP of the right eye was used as the right eye was also utilised for analysis of the retinal microvascular calibre immediately after IOP measurement.

\section{Biochemical analyses}

Sodium fluoride-, serum and whole blood EDTA samples were analysed for glucose, lipids, high sensitivity C-reactive protein (CRP), glycated haemoglobin $(\mathrm{HbAlC})$ and cotinine, $\gamma$-GT using the Cobas Integra 400 plus and Modular ROCHE Automated Analyser, Basel, Switzerland; as well as the Konelab ${ }^{\mathrm{TM}}$ 20I Sequential Multiple Analyser Computer, ThermoScientific, Vantaa, Finland respectively. Serum samples were analysed for total $\mathrm{T}$ using an electro chemiluminescence immunoassay on the Elecsys 2010 apparatus (Roche, Basel, Switzerland). Both the intra- and inter-assay coefficients of variation $(\mathrm{CV})$ for all the assays were less than $10 \%$. Free testosterone values were calculated according to the method of Vermeulen et al. [22].

\section{Statistical methods}

Data was analysed using Statistica ${ }^{\infty}$ software version 12.0 (Statsoft Inc.,
Tulsa, USA, 2012). Skewness of data were tested with the Shapiro-wilk test and $\gamma$-GT and CRP values were logarithmically transformed. Independent T-tests determined participant characteristic differences. $A$ priori covariates implicated in higher CVD risk were included [age, BSA, physical activity, dichotomised cotinine, $\log$ CRP and $\log \gamma$-GT] $[18,21]$. Chi-square $\left(\chi^{2}\right)$ statistics compared proportions. General linear model analyses, independent of $a$ priori co-variates, were computed to test interactions on main effects (race $\mathrm{x}$ total testosterone) and all potential cardiovascular risk markers (i.e. cholesterol, FT, pulse pressure and retinal microvasculature markers). As a result of the high correlation between CRAE and CRVE, CRAE was added as a covariate for CRVE and vice versa [23]. Independent t-tests comparisons were computed to compare 1) low and high testosterone male groups as well as 2) low testosterone black vs. white male groups.

Forward stepwise multiple regression analyses determined associations between retinal calibre and cardiometabolic markers in various models from two perspectives: firstly (model 1), in the total male sample, the dependent variables were AVR, CRVE and CRAE. Covariates included age, race, BSA, dichotomised cotinine, cholesterol, $\log$ CRP, $\log \gamma$-GT, physical activity, PP, FT, DOPP and CRAE (when CRVE was the dependent variable and vice versa). Secondly (model 2), the above regression analyses were repeated in stratified ethnic groups, excluding race as a covariate. The sample size of low testosterone groups was too small and multiple regression analyses could not be computed.

Sensitivity analyses: Forward stepwise regression analyses with similar dependent and independent covariates as described above, were repeated in several models in the total group as well as in the separate ethnic male 
Table I: Comparing mean $( \pm S D)$ unadjusted baseline characteristics of high and low testosterone African black and white men

\begin{tabular}{|c|c|c|c|}
\hline & $\begin{array}{l}\text { Low testosterone men } \\
\qquad(\mathrm{N}=\mathbf{7 2})\end{array}$ & $\begin{array}{l}\text { High testsoterone men } \\
\qquad(\mathbf{N}=\mathbf{8 1})\end{array}$ & $\mathbf{P}$ \\
\hline \multicolumn{4}{|l|}{ Lifestyle and biochemical variables } \\
\hline Age (years) & $46.2 \pm 7.51$ & $49.2 \pm 10.2$ & 0.03 \\
\hline Waist circumference $(\mathrm{cm})$ & $98.4 \pm 14.8$ & $106.1 \pm 13.1$ & $<0.001$ \\
\hline Body mass index (BMI) $\left(\mathrm{kg} / \mathrm{m}^{2}\right)$ & $28.3 \pm 5.64$ & $30.2 \pm 5.14$ & 0.02 \\
\hline Body surface area $\left(\mathrm{m}^{2}\right)$ & $1.98 \pm 0.23$ & $2.23 \pm 0.20$ & $<0.0001$ \\
\hline Physical activity (kcal/24h) & $3349.4 \pm 1247.9$ & $4049.9 \pm 1815.1$ & 0.003 \\
\hline Serum cholesterol $(\mathrm{mmol} / \mathrm{L})$ & $4.63 \pm 1.04$ & $4.20 \pm 1.00$ & 0.01 \\
\hline HDL cholesterol (mmol/L) & $0.93 \pm 0.34$ & $0.85 \pm 0.23$ & 0.05 \\
\hline Total cholesterol-to-HDL ratio & $5.42 \pm 1.86$ & $5.14 \pm 1.31$ & 0.24 \\
\hline Serum glucose $(\mathrm{mmol} / \mathrm{L})$ & $6.13 \pm 2.61$ & $4.70 \pm 1.27$ & $<0.0001$ \\
\hline HbA1C (\%) & $6.33 \pm 1.60$ & $5.69 \pm 0.86$ & 0.001 \\
\hline$\gamma-\mathrm{GT}, \mathrm{U} / \mathrm{L}$ & $79.3 \pm 84.6$ & $35.7 \pm 39.8$ & $<0.0001$ \\
\hline C-reactive protein $(\mathrm{mg} / \mathrm{L})$ & $6.02 \pm 11.8$ & $2.75 \pm 9.65$ & $<0.04$ \\
\hline Serum cotinine (\% smokers) & 25.0 & 12.2 & 0.03 \\
\hline \multicolumn{4}{|l|}{ Endocrine variables } \\
\hline Serum testosterone $(\mathrm{nmol} / \mathrm{L})$ & $13.8 \pm 6.51$ & $14.9 \pm 5.55$ & 0.22 \\
\hline Serum free testosterone $(\mathrm{nmol} / \mathrm{L})$ & $0.39 \pm 0.17$ & $0.34 \pm 0.12$ & 0.03 \\
\hline Serum bioavailable testosterone $(\mathrm{nmo} / \mathrm{L})$ & $7.99 \pm 3.21$ & $7.89 \pm 2.63$ & 0.81 \\
\hline \multicolumn{4}{|l|}{ Cardiovascular variables } \\
\hline 24h ABPM SBP (mm Hg) & $139 \pm 18$ & $128 \pm 11$ & $<0.0001$ \\
\hline 24h ABPM DBP (mm Hg) & $88 \pm 11$ & $79 \pm 8$ & $<0.0001$ \\
\hline 24h ABPM pulse pressure (mm Hg) & $51 \pm 10$ & $48 \pm 6$ & 0.02 \\
\hline Right IOP (mmHg) & $16.8 \pm 4.32$ & $14.8 \pm 3.25$ & $<0.001$ \\
\hline Right DOPP (mmHg) & $74.8 \pm 13.2$ & $71.8 \pm 10.3$ & 0.10 \\
\hline Retinal arteriole-to-venular ratio & $0.59 \pm 0.06$ & $0.63 \pm 0.04$ & $<0.0001$ \\
\hline Central retinal arterial equivalent (MU) & $147.9 \pm 13.2$ & $150.2 \pm 12.0$ & 0.25 \\
\hline Central retinal venular equivalent (MU) & $251.4 \pm 19.2$ & $237.9 \pm 18.5$ & $<0.0001$ \\
\hline Cardiovascular disease history, n (\%) & $4(5.56)$ & $25(20.3)$ & $<0.0001$ \\
\hline $\mathrm{HT}, \mathrm{n}(\%)(\mathrm{SBP} \geq 130$ and/or $\mathrm{DBP} \geq 80 \mathrm{~mm} \mathrm{Hg})$ & $56(78.9)$ & $39(48.1)$ & $<0.0001$ \\
\hline HT medication, $\mathrm{n}(\%)$ & $22(29.6)$ & $20(24.7)$ & 0.38 \\
\hline
\end{tabular}

groups, by a) excluding HIV-infected participants $(\mathrm{N}=16)$ and $\mathrm{b})$ adding physical activity, HT medication users, cotinine and/or serum glucose as independent covariates.

Mediation analyses: To assess the indirect effects of independent variables (FT and PP) on dependent variables (AVR, CRAE and CRVE), mediation analyses were performed with
IBM SPSS Statistics Version 22 (IBM Corporation) [24]. Significance was noted as $\mathrm{p} \leq 0.05$.

\section{Results}

Table I compares unadjusted baseline characteristics of black and white men. The black men displayed $(\mathrm{p}<0.01)$ lower physical activity and BSA compared to the white men. Furthermore, overall, they displayed a poorer cardiometabolic health profile than the white men with higher BP, hyperpulsatile pressure $(\mathrm{PP}>50 \mathrm{~mm}$ $\mathrm{Hg}$ ), resting levels of serum glucose, HbA1C and CRP. Remarkably, 79.8\% of the black men were hypertensive with $29.6 \%$ of the total group on HT 
Table II: Comparing mean ( \pm SD) unadjusted baseline characteristics of high and low testosterone men

\begin{tabular}{|c|c|c|c|}
\hline & $\begin{array}{l}\text { Low testosterone } \\
\text { men } \\
(\mathrm{N}=40)\end{array}$ & $\begin{array}{l}\text { High testosterone men } \\
\qquad(\mathrm{N}=143)\end{array}$ & $\mathbf{P}$ \\
\hline \multicolumn{4}{|l|}{ Cardiovascular variables } \\
\hline 24h ABPM SBP (mm Hg) & $140 \pm 17$ & $131 \pm 14$ & $<0.001$ \\
\hline 24h ABPM DBP (mm Hg) & $87 \pm 10$ & $82 \pm 10$ & $<0.01$ \\
\hline 24h ABPM pulse pressure (mm Hg) & $53 \pm 10$ & $49 \pm 7$ & $<0.01$ \\
\hline Right IOP (mmHg) & $15.9 \pm 4.01$ & $15.7 \pm 3.94$ & 0.83 \\
\hline Right DOPP (mmHg) & $76.3 \pm 11.6$ & $72.1 \pm 11.8$ & 0.04 \\
\hline Retinal arteriole-to-venular ratio & $0.59 \pm 0.05$ & $0.62 \pm 0.05$ & 0.003 \\
\hline Central retinal arterial equivalent (MU) & $147.5 \pm 13.1$ & $149.8 \pm$ & 0.31 \\
\hline Central retinal venular equivalent (MU) & $250.1 \pm 20.3$ & $242.4 \pm 19.7$ & 0.03 \\
\hline Serum testosterone $(\mathrm{nmol} / \mathrm{L}$ & $7.59 \pm 2.06$ & $16.79 \pm 4.99$ & $<0.001$ \\
\hline Free testosterone $(\mathrm{nmol} / \mathrm{L})$ & $0.22 \pm 0.07$ & $0.41 \pm 0.13$ & $<0.001$ \\
\hline & $\begin{array}{l}\text { Low testosterone } \\
\text { black men }\end{array}$ & $\begin{array}{c}\text { Low testosterone white } \\
\text { men }\end{array}$ & $\mathbf{P}$ \\
\hline & $(\mathrm{N}=25)$ & $(N=15)$ & \\
\hline \multicolumn{4}{|l|}{ Cardiovascular variables } \\
\hline 24h ABPM SBP (mm Hg) & $140 \pm 17$ & $131 \pm 15$ & $<0.001$ \\
\hline 24h ABPM DBP (mm Hg) & $87 \pm 10$ & $82 \pm 10$ & $<0.01$ \\
\hline 24h ABPM pulse pressure (mm Hg) & $53 \pm 10$ & $49 \pm 7$ & $<0.01$ \\
\hline Right IOP (mmHg) & $15.9 \pm 4.01$ & $15.7 \pm 3.94$ & 0.84 \\
\hline Right DOPP (mmHg) & $76.3 \pm 11.6$ & $72.1 \pm 11.8$ & 0.04 \\
\hline Retinal arteriole-to-venular ratio & $0.59 \pm 0.05$ & $0.62 \pm 0.05$ & 0.003 \\
\hline Central retinal arterial equivalent (MU) & $147.5 \pm 13.1$ & $149.8 \pm 12.4$ & 0.31 \\
\hline Central retinal venular equivalent (MU) & $250.1 \pm 20.3$ & $242.4 \pm 19.7$ & 0.03 \\
\hline Serum testosterone $(\mathrm{nmol} / \mathrm{L}$ & $7.45 \pm 2.16$ & $7.79 \pm 1.95$ & 0.60 \\
\hline Free testosterone (nmol/L) & $0.22 \pm 0.07$ & $0.23 \pm 0.06$ & 0.79 \\
\hline \multicolumn{4}{|c|}{$\begin{array}{l}\text { Data presented as unadjusted means with standard deviation with low testosterone defined as Total T } \leq 10.4 \mathrm{nmol} / \mathrm{L} \text {. BPM SBP, } 24 \mathrm{~h} \\
\text { ambulatory systolic blood pressure; ABPM DBP, } 24 \mathrm{~h} \text { ambulatory diastolic blood pressure; Right IOP, intra-ocular pressure of the right ey } \\
\text { Right DOPP, diastolic ocular perfusion pressure of the right eye; HT, hypertension; } 1 \mathrm{MU} \text {, measuring units, equal to } 1 \mu \mathrm{m} \text { in the normal } \\
\text { Gullstrand eye. }\end{array}$} \\
\hline
\end{tabular}

treatment compared to $48.1 \%$ of the white men who were hypertensive and $24.7 \%$ who were on treatment. The $\gamma$-GT levels of the black men were significantly higher than those of the white men, which may support other findings of high alcohol consumption in South African black men [16]. The serum $\mathrm{T}$ levels did not differ between the ethnic groups but the free testosterone (FT) levels of the black men were slightly higher than those of the white men.

Interactions adjusted for a priori covariates verified differences between ethnic male groups for DOPP
$\left(\mathrm{F}_{1,142}=3.66, \mathrm{p}=0.058\right)$ as well as AVR $\left(\mathrm{F}_{1,145}=8.39, \mathrm{p}=0.004\right)$. In Table II, comparisons between the total group of hypogonadic men with the normogonadic men showed that DOPP, CRVE and all of the BP variables, were higher and AVR lower in the hypogonadic men, whereas CRAE 
Table III: Forward stepwise regression analyses predicting relationships between the microvascular profiles, diastolic ocular perfusion pressure and testosterone

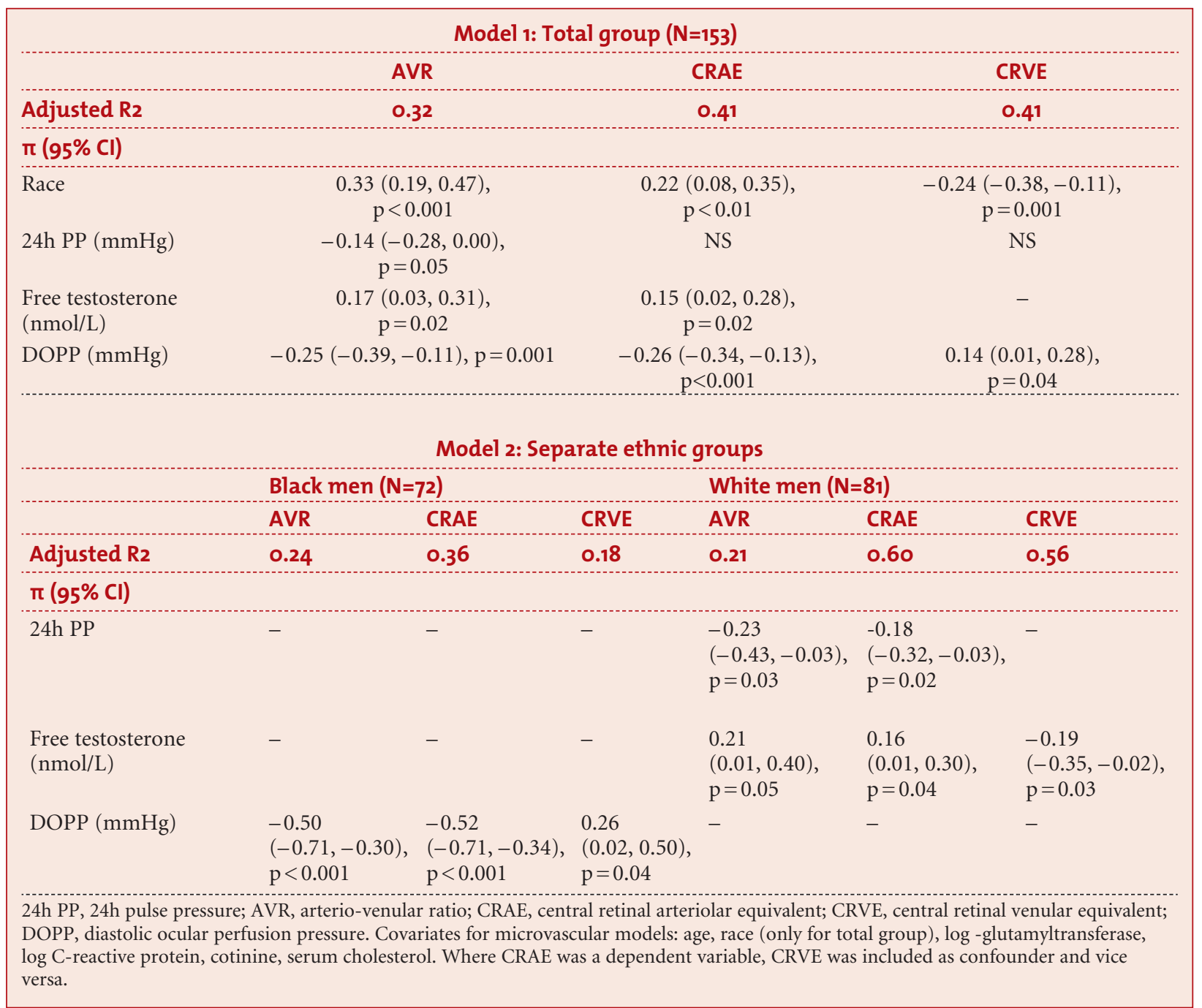

did not differ between the two groups. In the current sub study (Tab. IIB), there were 40 men ( 25 black and 15 white men) with total $\mathrm{T}$ values below $10.4 \mathrm{nmol} / \mathrm{l}$. Despite the fact that there were no differences in the total $\mathrm{T}$ and FT levels of these black and white hypogonadic men, SBP, DBP and CRVE were higher and AVR was lower in the black hypogonadic men compared to their white counterparts (Tab. IIB). There was no difference in DOPP, CRAE and PP values between the two groups.
Forward stepwise linear regression analyses (Tab. III) across the total bi-ethnic group demonstrated inverse associations of DOPP with $\operatorname{AVR}(\beta=-0.25, p=0.001)$, CRAE $(\beta=-0.26, p=0.001)$ and a positive association with CRVE $(\beta=0.14$, $\mathrm{p}=0.04)$. PP was also inversely associated with $\operatorname{AVR}(\beta=-0.14, p=0.05)$ and $\operatorname{CRAE}(\beta=-0.14, p=0.04)$ in the total cohort. FT was positively associated with AVR $(\beta=0.17$, $\mathrm{p}=0.02)$ and with CRAE $(\beta=0.15$, $\mathrm{p}=0.02)$. The stratified analysis by ethnicity revealed a different pattern for black and white men. In black men, DOPP was inversely associated with AVR $(\beta=-0.50, p<0.001)$ and CRAE $(\beta=-0.52, \mathrm{p}<0.001)$ respectively and positively associated with CRVE $(\beta=0.26, p=0.04)$. Neither PP nor FT entered the model in the black group. In the white subgroup, $\mathrm{PP}$ was inversely associated with $\operatorname{AVR}(\beta=-0.23, p=0.03)$ and CRAE $(\beta=-0.18, p=0.02)$. FT was positively associated with retinal calibre, i.e. $\operatorname{AVR}(\beta=0.21, \mathrm{p}=0.05)$ and CRAE $(\beta=0.16, p=0.04)$ and inversely associated with CRVE $(\beta=-0.19$, 
$\mathrm{p}=0.03)$. DOPP did not enter the model. In additional models, we entered the three independent variables (PP, FT and DOPP) one at a time, but this did not alter the significance of associations. When FT is mediated by PP to predict AVR, CRAE or CRVE, the Sobel test is not statistically significant $(\mathrm{p}=0.22)$. It implies that between $3.35 \%$ (CRVE) and $28 \%$ (CRAE) of the total effect may be due to the indirect effect of mediation. Sensitivity analyses showed that the significance of the models was not affected by variables such as physical activity, cotinine, serum glucose, HIV status or the use of HT medication.

\section{Discussion}

The main aim of this study was to determine whether $\mathrm{T}$ is independently associated with retinal vascular calibre or whether it plays only a secondary role via its effect on PP. The main findings revealed a positive association of FT with a healthier retinal calibre profile (larger arterioles and narrower venules) in the white men. This might suggest a protective effect of testosterone on the retinal vasculature, where an apparent vasodilatory response in the retinal resistance microvessels was observed in white men only. This association remained unchanged, whether DOPP and/or PP was included in the model, and may support the notion of an independent association between FT and retinal calibre. The association of FT with retinal microvascular calibre was absent in black men where DOPP was strongly associated with arteriolar narrowing and venular widening. As these indices relate to stroke risk, the latter finding might help to explain the previously reported increase in stroke risk of black men [5].

As a negative association of FT with $\mathrm{BP}$ variables has been reported [6] and increased BP is associated with a decrease in AVR and CRAE [5], the associations found in our study are in line with previous evidence. PP was shown to be associated with arterial function and, together with retinal perfusion pressure, is an important predictor of the progression of diabetic retinopathy $[19,20]$. We therefore decided to include PP as the BP covariate in our study. Furthermore, low DOPP has a very strong correlation with the development of open angle glaucoma, a disease whose development is strongly associated with insufficient ocular blood flow [8]. The association of FT with microvascular calibre could be the result of an independent effect of $\mathrm{T}$ on the microvessels or could be a secondary effect as result of the association of T with PP. These associations of FT with retinal microvascular calibre as well as the inverse association of PP with AVR and CRAE are also in accordance with what could be expected. Comparing the $\mathrm{BP}$ values of hypogonadic black with hypogonadic white men revealed that, despite the absence of a difference in the $\mathrm{T}$ values between the two groups, the BP values of black men were still higher than those of their white counterparts. This may suggest that the black men are more sensitive to the effect of low $\mathrm{T}$ on their vascular system.

DOPP may either be too low $(<50 \mathrm{mmHg})$, resulting in reduced perfusion and creating a risk for hypoxic damage, or if too high, creating a risk for ocular bleeding $[25,26]$. In general, the OPP (whether expressed as systolic OPP, mean arterial OPP or DOPP) is the driving force to ascertain sufficient blood flow through the ocular vasculature to provide nutrition to retinal neurons. OPP must therefore be adjusted on a continuous basis to correct for changes in the systemic BP. Blood flow in the ocular blood vessels is normally modified by changing the microvascular calibre since vessel diameter is the principal determinant of blood flow. Therefore, any factor associated with retinal vascular calibre, may also affect blood flow if OPP remains constant. In essential hypertension, increased DOPP via higher blood pressure and/or lower intraocular pressure, will enhance vascular resistance and reduce ocular blood flow to the optic nerve head, thereby inducing ischaemia [27]. This partially supports our findings in black men, where a higher risk for ischemia may be prevalent considering their mean BP values exceeding hypertensive status [18]. The fact that low compliance to hypertension treatment is evident might prove to be detrimental in the control of blood pressure and microvascular risk. It is known that serum $T$ is inversely associated with BP and positively associated with microvascular blood flow [28, 29 ]. This raises the possibility that reduced serum $T$ may be a complicating factor in pathologies associated with reduced perfusion in the retinal and, maybe, also other microvascular beds. However, very little data are available concerning the role of sex hormones in ocular blood flow [28].

A number of the role players determining retinal blood flow (PP, IOP and CRVE) were significantly higher in the black than in the white group. The adjusted value of DOPP was also higher in the black than in the white group but these differences were not reflected in the arteriolar calibre of the black men. However, the elevated DOPP values of the black men may have affected their retinal blood flow rate. Further studies are needed to clarify this matter.

The negative associations between DOPP (in the black men) and PP (in the white men) with AVR and CRAE supports the notion that increased PP (white men) and DOPP (black men) are associated with reduced retinal arteriolar calibre. The increased blood flow resulting from increased $\mathrm{BP}$ and DOPP may stimulate the autoregulatory mechanisms in the resistance ves- 
sels of the retinal microvasculature, thereby reducing the microvascular calibre to ultimately prevent excessive blood flow through the microvessels. In the white group, strong associations were found between serum FT and retinal microvascular calibre. This finding is in accordance with the results of Puttabyatappa et al. [11], who found that serum T mediates vasodilation in microvascular resistance vessels. Therefore, the positive association between FT and AVR/CRAE was to be expected in the white men who demonstrated lower PP values. Low T will, therefore, exert a vasoconstrictive effect on the microvasculature and is furthermore, also associated with increased PP [10]. Both low T and high PP are associated with reduced vasodilation, which may independently lead to reduced retinal arteriolar calibre. As venules are not resistance vessels, an increase in BP (associated with low $\mathrm{T}$ ) will tend to increase the venular calibre supporting the inverse association between FT and CRVE in the white men. Elimination of PP and/ or DOPP from this model did not affect the outcome of the analyses, supporting the notion that FT is independently associated with retinal microvascular calibre in the white men. This notion was also supported by a lack of significant mediation between FT and PP. These results were supported by similar independent associations between serum total $\mathrm{T}$ and retinal microvascular calibre (results not shown) Clinically, this finding may be of importance as attenuated serum $\mathrm{T}$ might lead to decreased ocular perfusion which is associated with ischemic damage, increased local metabolic demands, glaucoma development and loss of vision [30]. On a related note, it is well documented that an attenuated serum $\mathrm{T}$ level is associated with an increase in BP and other components of the metabolic syndrome like an increase in visceral fat and blood glucose [1]. The values of serum glucose, $\mathrm{HbA1C}$ and $\mathrm{PP}$ variables were significantly higher in black men than white men. In previous studies, we have found conspicuous differences between black and white groups in the effect of low $\mathrm{T}$ values and various variables $[3,4]$, which were notoriously difficult to explain. We cautiously speculate that $\mathrm{T}$ has an independent effect directly on the retinal vessels based on the fact that eliminating PP from the regression model did not change the associations of $\mathrm{T}$ with retinal microvascular calibre. T may also have a secondary influence on the retinal vessel calibre via its effect on $\mathrm{BP}$, which may induce a higher sensitivity to attenuated $\mathrm{T}$ in black men. This presumed higher sensitivity in black men may have played a role in die associations of attenuated $\mathrm{T}$ with various cardiovascular variables found in this and other studies [3, 4]. Despite the small sample size, we also found higher blood pressure and microvascular risk in black hypogonadic men when compared to their white counterparts.

Indeed, the inverse associations between PP and AVR and CRAE in the white group are in accordance with what could be expected [5], while DOPP, which is dependent on DBP, shows similar associations of reduced perfusion and retinal calibre changes in the black men. The mechanisms behind the differences in these relationships in the ethnic groups are not clear. More metabolic disturbances, higher BP, PP, and ocular perfusion pressure in black men may possibly have exerted a larger impact on ocular blood flow than $\mathrm{T}$ alone.

Several limitations of this study should be noted. Its cross-sectional design prevents causal inferences. A more representative and larger sample of the whole population of South Africa would be necessary to draw generalised conclusions.

\section{Conclusions}

FT was independently associated with retinal vascular calibre in white but not in black men from South Africa. High $\mathrm{PP}$ was associated with arteriolar narrowing in white men only, suggesting the possibility of an additional indirect association between $\mathrm{T}$ and retinal $\mathrm{mi}$ crovascular calibre. Reduced perfusion and lower ocular blood flow in black men may increase their ischaemia and vascular dysregulation, ultimately leading to an increase in their stroke risk. Further prospective studies are necessary to explain these differences between black and white men

\section{Acknowledgements}

The present work was partially supported by the North-West Department of Education, South African Medical Research Council, National Research Foundation, North-West University, ROCHE Diagnostics, South Africa, and the Metabolic Syndrome Institute, France. We gratefully acknowledge the voluntary collaboration of the participants. The SABPA study would not have been possible without the valuable contributions of co-investigators and technical staff.

\section{Sources of funding}

The present work was partially supported by the North-West Department of Education, South African Medical Research Council, National Research Foundation, North-West University, ROCHE Diagnostics, South Africa, and the Metabolic Syndrome Institute, France.

\section{Conflict of interest}

There is no conflict of interest existing. 


\section{References}

1 Jones TH. Testosterone deficiency: a risk factor for cardiovascular disease? Trends Endocrinol Metab 2010; 21: 496 - 503.

2 Toker E, Yenice Ö, Akpinar, I, et al. Influence of serum levels of sex hormones on intraocular pressure in menopausal women. J Glaucoma 2003; 12: $436-440$.

3 Malan NT, Hamer M, Schutte AE, et al. Low testosterone and hyperkinetic blood pressure responses in a cohort of South African men: the SABPA study. Clin Exp Hypertens 2012; 35: 228 - 235.

4 Malan NT, Hamer M, Lambert GW, et al. Sex hormones associated with subclinical kidney damage and atherosclerosis in South African men: the SABPA study. J Hypertens 2012; 30: 2387 - 2394.

5 Kawasaki R, Cheung N, Wang JJ, et al. Retinal vessel diameters and risk of hypertension: the Multi-ethnic Study of Atherosclerosis. J Hypertens 2009; 27 : 2386- 2393.

6 Henderson AD, Bruce BB, Newman NJ, et al. Hypertension-related eye abnormalities and the risk of stroke. Rev Neurol Dis 2011; 8: 1 -9.

7 Yanagi M, Kawasaki R, Wang JJ, et al. Vascular risk factors in glaucoma: a review. Clin Exp Ophthalmol 2011; 39: $252-258$.

8 Quaranta L, Gandolfo F, Turano R, et al. Effects of topical hypotensive drugs on circadian IOP, blood pressure, and calculated diastolic ocular perfusion pressure in patients with glaucoma. Invest Ophthalmol Vis Sci 2006; 47: $2917-2923$.

9 Jürgens C, Grossjohann R, Tost FHW. Relationship of systemic blood pressure with ocular perfusion pressure and intraocular pressure of glaucoma patients in telemedical monitoring. Med Sci Monit 2012; 18 : MT85-89.

10 Corona G, Manucci E, Lotti F, et al. Pulse pressure, an index of arterial stiffness, is associated with androgen deficiency and impaired penile blood flow in men with ED. J Sex Med 2009; 6: 285 - 293.

11 Puttabyatappa Y, Stallone JN, Ergul A, et al. Peroxynitrite mediates testosteroneinduced vasodilation of microvascular re- sistance vessels. J Pharmacol Exp Ther 2013; 345: 7 - 14 .

12 Malan L, Hamer M, Frasure-Smith N, et al. COHORT PROFILE: Sympathetic activity and Ambulatory Blood Pressure in Africans (SABPA) Prospective Cohort Study. Int J Epidem 2014. doi: 10.1093/ije/dyu199.

13 Opie LH, Seedat YK. Hypertension in Sub-Saharan populations. Circulation 2005; 112: $3562-3568$.

14 Jarvis MJ, Feyerabend C, Bryant A, et al. Passive smoking in the home: plasma cotinine concentrations in non-smokers with smoking partners. Tob Control 2001; 10 : $368-74$.

15 Zielinska-Danch W, Wardas W, Sobczak A, Szoltysek-Boldys I. Estimation of urinary cotinine cut-off points distinguishing non-smokers, passive smokers and active smokers. Biomarkers; 12 (5): 484 - 496.

16 Zatu MC, van Rooyen JM, Loots du T, et al. A comparison of the cardiometabolic profile of black South Africans with suspected non-alcoholic fatty liver disease (NAFLD) and excessive alcohol use. Alcohol 2015; 49 (2): $165-72$

17 Mosteller RD. Simplified calculation of body-surface area. N Eng J Med 1987; 317: 1098.

18 Mancia G, Fagard R, Narkiewicz K, et al. 2013 ESH/ESC Guidelines for the management of arterial hypertension. The Task Force for the management of arterial hypertension of the European Society of Hypertension (ESH) and of the European Society of Cardiology (ESC). J Hypertens 2013; 31: $1281-1357$.

19 Hayman DM, Xiao Y, Yao Q, et al. Alterations in pulse pressure affect artery function. Cell Mol Bioeng 2012; 5: 474-487.

20 Patel V, Sailesh S, Panja S, et al. Retinal perfusion pressure and pulse pressure: clinical parameters predicting progression to sight-threatening diabetic retinopathy. Br J Diabetes Vasc Dis 2001; 1: 80 - 87.

21 Knudtson MD, Lee KE, Hubbard LD, et al. Revised formulas for summarizing retinal vessel diameters. Curr Eye Res 2003; 27 : $143-149$.

22 Vermeulen A, Verdonck L \& Kaufman JM. A critical evaluation of simple methods for the estimation of free testosterone in serum. J Clin Endocrin Metab; 84 (10): 3666-3672
23 Nguyen TT, Wang JJ, Sharrett AR, et al. Relationship of retinal vascular calibre with diabetes and retinopathy. Diabetes Care 2008; 31: 544-549.

24 Preacher KJ, Hayes AF. Asymptotic and resampling strategies for assessing and compared indirect effects in multiple mediator models. Behav Res Methods 2008; 40 (3): 879 - 891

25 Cherecheanu AP, Garhofer G, Schmidl D, et al. Ocular perfusion pressure and ocular blood flow in glaucoma. Curr Opin Pharmacol 2013; 13: 36-42

26 Lovasik JV, Kergoat H. Systemic determinants. In Ocular blood flow, $1^{\text {st }} \mathrm{Ed}$. Ed: Schmetterer L, Kiel JW. 2012:173-184. Springer Verlag, Berlin Heidelberg.

27 Flammer, J.;Konieczka, K.;Flammer, A.J.; The primary vascular dysregulation syndrome: implications for eye diseases. EPMA Journal 2013;4:14. doi:10.1186/1878-5085-4-14.

28 Schmidl D, Schmetterer L, Garhöfer $\mathrm{G}$, et al. Gender differences in ocular blood flow. Current Eye Res 2015; 40 (2): $201-212$.

29 Kelly DM, Jones TH. Testosterone: a vascular hormone in health and disease. J Endocrinol 2013; 217: R47 - R71.

30 Moore D, Harris A, WuDunn D. Dysfunctional ocular blood flow: a risk factor for glaucoma? Clin Ophthalmol 2008; 2 (4): $849-861$.

\section{Correspondence address}

\section{Dr. Nico T. Malan}

Hypertension in Africa Research

Team

Faculty of Health Sciences

North-West University

Hoffman Street, Private Bag X6001

Potchefstroom 2520

South Africa

nico.malan@nwu.ac.za

Submitted: 26.03.2015

Accepted after revision: 07.05.2015 